\title{
Prevalence and Spectrum of In Utero Structural Brain Abnormalities in Fetuses with Complex Congenital Heart Disease
}

\author{
M. Brossard-Racine, A.J. du Plessis, G. Vezina, R. Robertson, D. Bulas, I.E. Evangelou, M. Donofrio, D. Freeman, and C. Limperopoulos
}

\begin{abstract}
BACKGROUND AND PURPOSE: Brain injury is a major complication in neonates with complex congenital heart disease. Preliminary evidence suggests that fetuses with congenital heart disease are at greater risk for brain abnormalities. However, the nature and frequency of these brain abnormalities detected by conventional fetal MR imaging has not been examined prospectively. Our primary objective was to determine the prevalence and spectrum of brain abnormalities detected on conventional clinical MR imaging in fetuses with complex congenital heart disease and, second, to compare the congenital heart disease cohort with a control group of fetuses from healthy pregnancies.
\end{abstract}

MATERIALS AND METHODS: We prospectively recruited pregnant women with a confirmed fetal congenital heart disease diagnosis and healthy volunteers with normal fetal echocardiogram findings who underwent a fetal MR imaging between 18 and 39 weeks gestational age.

RESULTS: A total of 338 fetuses (194 controls; 144 with congenital heart disease) were studied at a mean gestational age of $30.61 \pm 4.67$ weeks. Brain abnormalities were present in $23 \%$ of the congenital heart disease group compared with $1.5 \%$ in the control group $(P<.001)$. The most common abnormalities in the congenital heart disease group were mild unilateral ventriculomegaly in $12 / 33$ ( $36.4 \%)$ and increased extra-axial spaces in 10/33 (30.3\%). Subgroup analyses comparing the type and frequency of brain abnormalities based on cardiac physiology did not reveal significant associations, suggesting that the brain abnormalities were not limited to those with the most severe congenital heart disease.

CONCLUSIONS: This is the first large prospective study reporting conventional MR imaging findings in fetuses with congenital heart disease. Our results suggest that brain abnormalities are prevalent but relatively mild antenatally in fetuses with congenital heart disease. The long-term predictive value of these findings awaits further study.

ABBREVIATIONS: $C H D=$ congenital heart disease; $G A=$ gestational age; $H L H S=$ hypoplastic left-heart syndrome

B rain injury is a major complication in neonates with complex congenital heart disease (CHD). ${ }^{1,2}$ During the past decade, a growing body of evidence has demonstrated the high prevalence

Received November 20, 2013; accepted after revision December 27.

From the Advanced Pediatric Brain Imaging Research Laboratory (M.B.-R., I.E.E., D.F., C.L.), Division of Diagnostic Imaging and Radiology (M.B.-R., G.V., D.B., I.E.E., D.F.,

C.L.), Fetal and Transitional Medicine (M.B.-R., A.d.P., M.D., C.L.), and Division of Cardiology (M.D.), Children's National Medical Center, Washington DC; and Department of Radiology (R.R.), Children's Hospital Boston and Harvard Medical School, Boston, Massachusetts.

This study is supported by the Canadian Institutes of Health Research (MOP-81116); Marie Brossard-Racine was financially supported by the Fonds de Recherche en

Sante du Quebec postdoctoral fellowship at the time of manuscript preparation. Paper previously presented at: Annual Meeting of the Pediatric Academic Societies, May 4-7, 2013; Washington, DC

Please address correspondence to Catherine Limperopoulos, PhD, Advanced Pediatric Brain Imaging Research Laboratory, Division of Diagnostic Imaging and Radiology/Fetal and Transitional Medicine, Children's National Medical Center, 111 Michigan Ave NW, Washington D.C., 20008; e-mail: climpero@childrensnational.org - Indicates open access to non-subscribers at www.ajnr.org

http://dx.doi.org/10.3174/ajnr.A3903 of preoperative brain imaging abnormalities in neonates with $\mathrm{CHD}$, suggesting an antenatal origin in many cases. ${ }^{3-6}$ More recently, our group has used quantitative MR imaging to demonstrate a progressive third-trimester deceleration in volumetric brain growth and metabolism in fetuses with $\mathrm{CHD},{ }^{7}$ reduced volumes of gray (cortical and subcortical) and white matter, and significant delays in cortical gyrification and surface area in fetuses with hypoplastic left-heart syndrome (HLHS). ${ }^{8}$ To date, there is only 1 published report of brain findings in fetuses with CHD by using conventional MR imaging. ${ }^{9}$ This retrospective chart review of 53 cases reported a 39\% incidence of brain anomalies in fetuses with CHD. Taken together, preliminary evidence suggests that fetuses with CHD are at risk of brain abnormalities, including delayed maturation and brain injury. However, to date, no study has prospectively ascertained the frequency and nature of brain abnormalities by conventional MR imaging in fetuses with CHD. The primary objective of this study was to determine 
the prevalence and spectrum of brain abnormalities detected on conventional clinical MR imaging in fetuses with complex CHD and, second, to compare the CHD cohort with a control group of fetuses from healthy pregnancies.

\section{MATERIALS AND METHODS \\ Subjects}

As part of an ongoing prospective observational study that started in September 2007, pregnant women with a fetal CHD diagnosis confirmed by echocardiography at Boston Children's Hospital ${ }^{7}$ and Children's National Medical Center were recruited as patients. A fetal echocardiography was performed by one of the center's experienced fetal echocardiographers on the day of the mother's appointment. Fetal CHD diagnoses were subsequently categorized as cyanotic-versus-acyanotic and single- versus 2 -ventricle physiology as determined by oxygen saturation and anatomic classification. During the same study timeframe, healthy controls were also recruited from healthy volunteers with normal fetal echocardiography findings or pregnant women for whom an echocardiography was performed for a previous family history of CHD. Patients and controls with multiple pregnancies, abnormalities on fetal sonography, congenital infection, documented chromosomal abnormalities, and/or multiorgan dysmorphic conditions were excluded from the study. ${ }^{7}$ Thereafter, enrolled pregnant women completed a fetal MR imaging during the second or third trimester of pregnancy. Additionally, we amended our original protocol in 2013 to include serial fetal MR imaging studies (ie, 2 fetal MR imaging studies) as part of this prospective study, to examine fetal brain development during the second and third trimesters. For the subset of fetuses that underwent 2 fetal MR imaging scans, both studies were included in our analyses. Clinical and demographic information for each subject was collected through medical record reviews or questionnaires. Written informed consent was obtained for every participant, and the study was approved by the institutional review boards of Boston Children's Hospital and Children's National Medical Center.

\section{MR Imaging Data Acquisition}

Each fetal MR imaging study was performed on a $1.5 \mathrm{~T}$ scanner either on an Achieva (Philips Healthcare, Best, Netherlands) device by using a 5-channel phased-array coil (Boston Children's cohort) or on a Discovery MR450 scanner (GE Healthcare, Milwaukee, Wisconsin) by using an 8-channel phased-array coil (Children's National Medical Center cohort). Multiplanar singleshot fast spin-echo imaging sequences were performed as follows: on the Philips scanner, single-shot fast spin-echo TE $=120 \mathrm{~ms}$, TR $=971 \mathrm{~ms} /$ section, 330-mm FOV, $256 \times 204$ acquisition matrix; for the GE scans, TE $=160 \mathrm{~ms}$, TR $=1110 \mathrm{~ms} / \mathrm{section}$, 320-mm FOV, $256 \times 192$ acquisition matrix. Diffusion tensor imaging was acquired in 12 directions with the following parameters: $\mathrm{TE}=$ minimum, $\mathrm{TR}=8000 \mathrm{~ms}$, and $4-\mathrm{mm}$ section thickness. The FOV was $330 \mathrm{~mm}$ on the GE scanner versus $280 \mathrm{~mm}$ on the Philips scanner. Finally, a T1 spoiled gradient-recalled acquisition was performed with $\mathrm{TE}=$ minimum, $\mathrm{TR}=100 \mathrm{~ms} / \mathrm{section}$, 360 -mm FOV , $256 \times 160$ acquisition matrix on the GE scanner. No contrast or sedation was used for any of the MR imaging studies.
All MR imaging studies were reviewed for clinical findings by one of the center's expert pediatric neuroradiologists, both of whom had $>15$ years of experience with fetal brain MR imaging (R.R. at Boston Children's Hospital and G.V. at Children's National Medical Center). The neuroradiologists were blinded to patient-versus-control status, the subject's medical history, and type of CHD. All studies were reviewed for the presence of developmental malformations, acquired abnormalities, and brain maturation following the clinical guidelines in place at the time of the study. Mild ventriculomegaly was diagnosed when the maximum width of the lateral ventricles at the level of the atrium measured between 10 and $15 \mathrm{~mm} .{ }^{10}$ Increased extra-axial space was diagnosed when the distance between the inner margin of the cranium and the outer margin of the frontal, temporal, parietal, and/or occipital regions was considered enlarged for gestational age (GA). ${ }^{11,12}$ Finally, images were also examined for signal abnormality on T2/T1 images and the presence of restricted diffusion on diffusion imaging acquisitions.

\section{Statistical Analysis}

Descriptive statistics by using means, SDs, and frequencies were used to characterize the sample and the nature of the brain abnormalities described on conventional MR imaging. Independent sample $t$ tests and $\chi^{2}$ analyses were used to explore differences and associations between groups, $\mathrm{CHD}$ diagnoses, cyanotic $\mathrm{CHD}$ versus acyanotic, single- versus 2-ventricle physiology, sex, GA, and MR imaging abnormalities. All analyses were performed by using the Statistical Package for the Social Sciences, Version 20 (IBM, Armonk, New York).

\section{RESULTS}

\section{Group Characteristics}

A total of 338 pregnant women (144 with fetuses with CHD, 194 controls) successfully completed second- or third-trimester MR imaging. Of these fetuses, 182 (53.8\%) were male ( 82 with CHD, 100 controls). As described in our "Materials and Methods" section, in 2013, a second fetal MR imaging was added to our MR imaging protocol (ie, 2 fetal MR imaging studies) to examine fetal brain development serially. Consequently, 28 women (10 fetuses with CHD, 28 controls) completed 2 fetal MR imaging studies, the first during the second trimester and the second during the third trimester, resulting in $376 \mathrm{MR}$ imaging studies. The mean GA of these 376 studies was $30.61 \pm 4.67$ weeks (range, 18.29-39.29 weeks), of which 273 (72.6\%) were performed during the third trimester. Although a significantly smaller proportion of studies was performed during the second trimester in the control group compared with the CHD group $\left(\chi^{2}=12.75, P<.001\right)$, the mean GA at MR imaging for the control group (29.94 \pm 5.14 weeks) was significantly lower compared with that in the CHD group (31.57 \pm 3.71 weeks; $t=3.57 ; P<.001)$.

The most common CHD diagnostic categories in our sample were the following: HLHS (32/144, 22.2\%), transposition of the great arteries $(24 / 144,18.8 \%)$, pulmonary atresia $(12 / 144,8.3 \%)$, tetralogy of Fallot $(17 / 144,11.8 \%)$, and double-outlet right ventricle $(16 / 144,11.1 \%)$. The complete distribution of fetal CHD diagnoses is presented in Table 1. 


\section{Abnormal Conventional MR Imaging Findings}

Brain abnormalities detected on MR imaging were significantly more frequent among patients with $\mathrm{CHD}\left(\chi^{2}=36.87, P<.001\right)$ and were detected in approximately one-quarter $(33 / 144,22.9 \%)$ of the fetuses with CHD compared with only 1.5\% (3/194) in the controls. The type and distribution of brain abnormalities on fetal MR imaging in the CHD group are summarized in Table 2. Mild unilateral ventriculomegaly (Fig 1) was the most common abnormality reported in $8.3 \%(12 / 144)$. Of these cases, left-sided ventriculomegaly was twice as common $(n=8)$ as right-sided $(n=$ 4). Ventriculomegaly was isolated in all cases, with the exception of 2 fetuses, one of which also presented with increased extra-axial CSF spaces and the second with a frontal subependymal cyst. Only 1 fetus presented with mild symmetric ventriculomegaly. The presence of increased extra-axial CSF spaces (Fig 2) was the second most common MR imaging abnormality in our cohort, reported in $6.9 \%(10 / 144)$ of CHD subjects. Three fetuses $(2.1 \%)$ with $\mathrm{CHD}$ were diagnosed with isolated inferior vermian hypoplasia on MR imaging (ie, no other associated cerebral malformations were identified). Three other fetuses $(2.1 \%)$ presented with immature brain appearance defined as an underdevelopment of the anatomic structures, such as fissures and sulci being less developed than expected for the estimated GA. Finally 4 patients presented with white matter abnormalities, which included cysts in 2 fetuses (1.4\%) and signal hyperintensity on T2-weighted

Table 1: CHD diagnoses distribution $(n=144)$

\begin{tabular}{lcc}
\hline \multicolumn{1}{c}{ Diagnosis } & Counts (\%) & $\begin{array}{c}\text { Abnormal MRI } \\
\text { Findings (\%) }\end{array}$ \\
\hline Hypoplastic left-heart syndrome & $32(22.2)$ & $7(21.21)$ \\
Transposition of the great artery & $27(18.8)$ & $5(15.2)$ \\
Double-outlet right ventricle & $16(11.1)$ & $5(15.2)$ \\
Tetralogy of Fallot & $17(11.8)$ & $5(15.2)$ \\
Pulmonary atresia & $12(8.3)$ & $1(3.0)$ \\
Atrioventricular septal defect & $7(5.9)$ & $4(12.1)$ \\
Aortic stenosis & $6(4.2)$ & $2(6.1)$ \\
Tricuspid atresia & $6(4.2)$ & $0(0.0)$ \\
Ventricular septal defect & $4(2.8)$ & $0(0.0)$ \\
Hypoplastic right-heart syndrome & $4(2.8)$ & $1(3.0)$ \\
Pulmonary stenosis & $2(1.4)$ & $1(3.0)$ \\
Truncus arteriosus & $2(1.4)$ & $2(6.1)$ \\
Ebstein anomaly & $1(0.7)$ & $0(0.0)$ \\
Severe coarctation of the aorta & $1(0.7)$ & $0(0.0)$ \\
Total & $144(100)$ & $33(100)$ \\
\hline
\end{tabular}

imaging in 2 others (1.4\%), one of which also demonstrated globally decreased diffusion in the white matter.

The 3 brain abnormalities detected in the control group included the following: right-sided mild unilateral ventriculomegaly $(0.5 \%)$, left germinal matrix hemorrhage $(0.5 \%)$, and mild cerebral underdevelopment $(0.5 \%)$. A fourth fetus had $2 \mathrm{D}$ brain biometrics that were slightly less than expected for GA on a second-trimester MR imaging; however, these normalized on a third-trimester MR imaging, and the brain was considered normal. As indicated in our inclusion criteria, no brain abnormalities were previously reported on fetal sonography in our cohort.

\section{Fetal Brain Anomalies and Type of CHD}

There was no significant association between type of brain abnormality and CHD diagnosis, GA at MR imaging, or sex. Unilateral ventriculomegaly was more frequent in the fetuses with HLHS (5/12, 41.7\%), while increased extra-axial CSF spaces were more common in fetuses with a double-outlet right ventricle $(3 / 10$, $30.0 \%)$. Of the 3 fetuses with isolated inferior vermian hypoplasia, the first had HLHS, the second was diagnosed with tetralogy of Fallot, and the third had truncus arteriosus. One fetus with tetralogy of Fallot presented with cysts in the periventricular white matter, and another fetus with transposition of the great arteries demonstrated isolated left-frontal subependymal cysts. Immature brain appearance was reported in 3 fetuses, one with tetralogy of Fallot, the second with double-outlet right ventricle, and the third with transposition of the great arteries. Finally, in 2 fetuses with transposition of the great arteries, there was evidence of diffuse white matter hyperintensity in the first and a focal hyperintense signal in the deep frontal white matter in the second.

Subgroup analyses based on cardiac physiology (ie, singleversus 2-ventricle physiology and cyanotic-versus-acyanotic lesions) were performed, but no significant association was found $\left(\chi^{2}=1.91, P=.662\right)$. However, increased extra-axial CSF spaces were primarily diagnosed in fetuses with acyanotic heart disease (7/10), while unilateral ventriculomegaly was 3 times more frequent in fetuses with cyanotic CHD. White matter signal abnormalities, cysts, and vermian hypoplasia were exclusively described in the subgroup with cyanotic lesions. The distribution of brain abnormalities between fetuses with cyanotic-versus-acyanotic CHD is reported in Table 3.

Table 2: Abnormal brain MRI findings per congenital heart disease diagnoses $(n=33)$

\begin{tabular}{|c|c|c|c|c|c|c|c|}
\hline $\begin{array}{c}\text { CHD } \\
\text { Diagnosis }\end{array}$ & Ventriculomegaly & $\begin{array}{c}\text { Increased Extra-Axial } \\
\text { Spaces }\end{array}$ & $\begin{array}{c}\text { Vermis } \\
\text { Hypoplasia }\end{array}$ & Cysts & $\begin{array}{c}\text { WM Signal } \\
\text { Hyperintensity }\end{array}$ & $\begin{array}{c}\text { Immature } \\
\text { Appearance }\end{array}$ & Total \\
\hline HLHS & 5 & 1 & 1 & - & - & - & 7 \\
\hline TGA & 1 & - & - & 1 & 2 & 1 & 5 \\
\hline TOF & 2 & - & 1 & 1 & - & 1 & 5 \\
\hline DORV & 1 & 3 & - & - & - & 1 & 5 \\
\hline AS & 1 & 1 & - & - & - & - & 2 \\
\hline AV canal SD & 2 & 2 & - & - & - & - & 4 \\
\hline PA & - & 1 & - & - & - & - & 1 \\
\hline PS & 1 & - & - & - & - & - & 1 \\
\hline $\mathrm{HRH}$ & - & 1 & - & - & - & - & 1 \\
\hline Truncus arteriosus & - & 1 & 1 & - & - & - & 1 \\
\hline Total & 13 & 10 & 3 & 2 & 2 & 3 & 33 \\
\hline
\end{tabular}

Note:-TGA indicates transposition of the great arteries; TOF, tetralogy of Fallot; DORV, double-outlet right ventricle; AS, aortic stenosis; AV canal SD, atrioventricular septal defect; PA, pulmonary atresia; PS, pulmonary stenosis; $\mathrm{HRH}$, hypoplastic right-heart syndrome. 

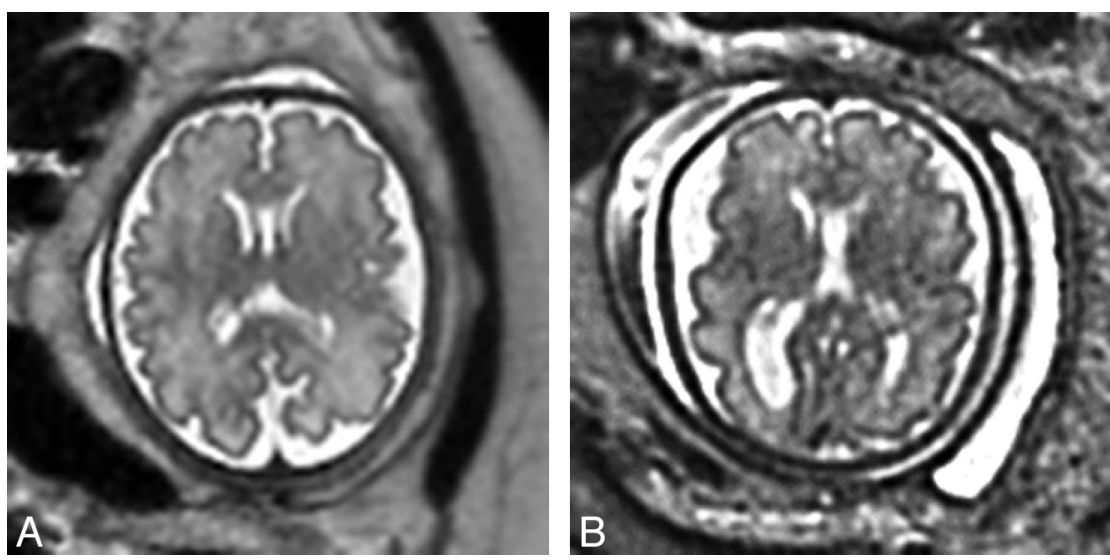

In the control group, the 3 brain abnormalities were noted on second-trimester MR imaging studies. The fetus with a germinal matrix hemorrhage in the left ventricle underwent a second MR imaging during the third trimester, and mild enlargement of the lateral ventricle secondary to the hemorrhage was reported.

\section{DISCUSSION}

This is the first prospective study to describe the prevalence of brain abnormalities by conventional clinical MR imag-

FIG 1. A, T2-weighted axial view of the brain of a healthy control at 32.55 weeks' GA. B, T2weighted axial view of the brain of a patient with unilateral left ventriculomegaly at 32.00 weeks' GA.
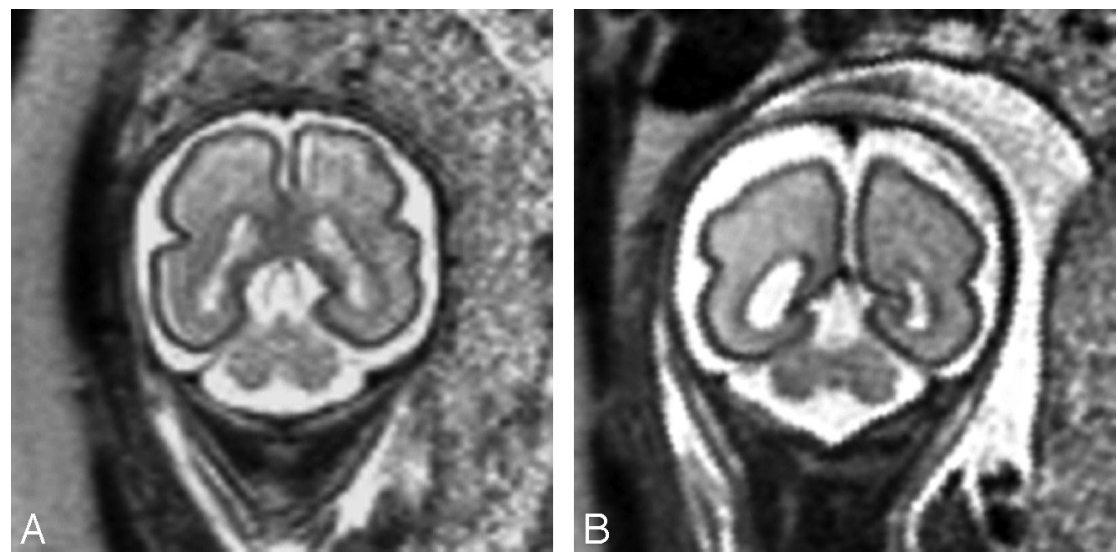

FIG 2. A, T2-weighted axial view of the brain of a healthy control at 26.56 weeks' GA. B, T2weighted axial view of the brain of a patient with extra-axial spaces at 27.28 weeks' GA.

ing in a large, consecutive cohort of fetuses with $\mathrm{CHD}$ and to compare them with a control group of fetuses from healthy pregnancies. We found that structural brain abnormalities were significantly more frequent in fetuses with CHD, with approximately one-quarter (23\%) demonstrating in utero structural brain abnormalities compared with $<2 \%$ in the control group. Mild unilateral ventriculomegaly and increased extra-axial CSF spaces were most commonly reported, together representing almost $70 \%(22 / 33)$ of all antenatal brain abnormalities in fetuses with CHD. Other findings among fetuses with CHD included vermian hypoplasia $(n=3)$, cerebral cortical immaturity $(n=3)$, periventricular cysts $(n=2)$, white matter signal hyperintensity $(n=$

Table 3: Distribution of abnormal brain MRI findings in cyanoticversus-acyanotic fetuses with CHD $(n=33)$

\begin{tabular}{lccc}
\hline \multicolumn{1}{c}{ Brain Abnormalities } & Acyanotic & Cyanotic & Total \\
\hline Increased extra-axial spaces & 7 & 3 & 10 \\
Ventriculomegaly & 4 & 9 & 13 \\
Vermis hypoplasia & 0 & 3 & 3 \\
Immature appearance & 1 & 2 & 3 \\
White matter signal hyperintensity & 0 & 2 & 2 \\
Cysts & 0 & 2 & 2 \\
Total & 12 & 21 & 33 \\
\hline
\end{tabular}

\section{Fetal Brain Abnormalities and Gestational Age}

The mean GA of fetuses with CHD with brain abnormalities was $31.15 \pm 3.46$ weeks and was not significantly different compared with fetuses with CHD with normal findings on brain MR imaging studies (mean GA $=31.78 \pm 3.78$ weeks; $t=0.748, P=.44$ ). Abnormalities were not found to be significantly more frequent during the second or third trimester $\left(\chi^{2}=1.91, P=.662\right)$, and their distribution is reported in Table 4. Among the $35 \mathrm{MR}$ imaging studies with abnormal findings in the CHD group, 2 fetuses were scanned twice (ie, in the second and third trimesters). One fetus presented with mild bilateral ventriculomegaly, while the second presented with immature brain appearance. For both fetuses, findings remained consistent at both MR imaging time points.
$2)$, and mild bilateral ventriculomegaly $(n=1)$. Finally, we found no significant difference in the prevalence of brain abnormalities in fetuses with single- versus 2-ventricle physiology or in cyanotic-versus-acyanotic lesions.

Unilateral ventriculomegaly was found in 12 (8\%) of the fetuses with CHD. Our findings corroborate the retrospective findings reported by Mlczoch et al, ${ }^{9}$ who described mild asymmetric dilation of the lateral ventricles as the most common brain anomaly in this population. Unilateral mild ventriculomegaly is, in fact, one of the most common brain abnormalities referred for fetal MR imaging and counseling in clinical fetal centers ${ }^{11,13}$ and is estimated to affect $0.07 \%$ of all pregnancies. ${ }^{10}$ Only 1 fetus in our control group $(0.05 \%)$ presented with mild unilateral ventriculomegaly.

Increased extra-axial CSF spaces were uniquely reported in fetuses with CHD and were present in 10 cases ( 9 reported in the third trimester), making this the second most common abnormality in this group. Enlarged extra-axial CSF spaces were mild and isolated in all cases, except in 1 fetus that also had mild unilateral ventriculomegaly. The finding of increased extra-axial CSF spaces could be due to delayed parenchymal brain development or disturbed CSF dynamics (possibly due to the fetal cardiac anomaly) or both. Additionally, immature cerebral cortical development was detected in 2 fetuses with CHD (2\%) compared with 
Table 4: Distribution of abnormal brain MRI findings among fetuses with CHD per trimester of pregnancy $(n=35)^{a}$

\begin{tabular}{lccc}
\hline \multicolumn{1}{c}{ Brain Abnormalities } & $\begin{array}{c}\text { Second } \\
\text { Trimester }\end{array}$ & $\begin{array}{c}\text { Third } \\
\text { Trimester }\end{array}$ & Total \\
\hline Increased extra-axial spaces & 1 & 9 & 10 \\
Ventriculomegaly & 4 & 10 & 14 \\
Vermian hypoplasia & 0 & 3 & 3 \\
Immature appearance & 2 & 2 & 4 \\
White matter signal hyperintensity & 0 & 2 & 2 \\
Cysts & 0 & 2 & 2 \\
Total & 7 & 28 & 35 \\
\hline
\end{tabular}

a Two fetuses were scanned twice, once at each trimester. One had immature brain appearance, and the other had bilateral ventriculomegaly. Both anomalies were detected at the second-trimester MRI and persisted at the third-trimester MRI.

only $1(0.5 \%)$ in the control group. Together, these findings support the notion that delayed brain maturation is likely a direct consequence of the cardiac defect. In our previous studies of fetuses with $\mathrm{CHD}$ by using quantitative brain MR imaging techniques, we described a progressive third-trimester deceleration in cerebral growth ${ }^{7}$ and delayed cortical maturation (in fetuses with HLHS $)^{8}$ compared with healthy fetuses. In the current study, 3 of these cases of suspected delay in brain maturation were detected by clinical MR imaging readings before the third trimester. Ten women in our CHD sample underwent repeated fetal MR imaging studies in the second and third trimesters, and in all cases, the findings were enduring within cases. Nevertheless, a larger sample with serial fetal MR imaging studies is needed to better delineate the onset and progression of delayed brain development in fetuses with CHD.

Disturbances in oxygen/substrate supply present a major threat to brain development in fetuses with CHD. ${ }^{14,15}$ Using Doppler sonography, Donofrio et $\mathrm{al}^{15}$ compared the cerebralplacental ratio between fetuses with $\mathrm{CHD}$ and healthy controls and showed that the normal intrinsic cerebral compensatory response to insufficient oxygen-substrate delivery (ie, cerebral vasodilation) was activated in fetuses with CHD. The cerebral-placental ratio was significantly lower overall in fetuses with CHD, with values $<1.0$ most prevalent in fetuses with a single-ventricle physiology. Reduced cerebral-placental ratio was significantly associated with decreased head circumference, supporting the notion that the "brain-sparing" response was insufficient to sustain brain growth. In addition, we have previously reported a significant association between smaller total brain volume and lower combined ventricular output from the aorta in fetuses with CHD. ${ }^{7}$ In the current study, evidence of brain abnormalities, including delayed brain development, was present among fetuses with various CHD diagnoses, suggesting that the risk of disturbed brain maturation in fetuses with CHD is not restricted to those with the most complex cardiac lesions, such as HLHS. This is also consistent with Mlczoch et al, ${ }^{9}$ who found no association between brain abnormalities and the type of CHD diagnosis. Future studies that relate brain abnormalities to specific Doppler ultrasound measures are needed to address this important question.

One fetus in the control group had a germinal matrix hemorrhage detected on MR imaging at 23 weeks' GA. Germinal matrix hemorrhage is a common complication of premature birth but has been rarely described in the fetus in utero. The history of the pregnancy was unremarkable. Secondary enlargement of the af- fected ventricle was reported on the follow-up MR imaging at 32 weeks and was consistent with the few in vivo cases reported in the literature. ${ }^{16}$ The patient was referred for clinical neurologic consultation and follow-up.

Three fetuses in our CHD cohort were diagnosed with inferior vermian hypoplasia, an anomaly that may represent delayed or arrested development of the cerebellar vermis. In previous studies, we have described an overall favorable prognosis for infants and school-aged children with a fetal MR imaging diagnosis of isolated vermian hypoplasia. ${ }^{17}$ However, the impact of inferior vermian development on subsequent neuropsychologic development in fetuses with CHD is unknown and awaits further investigation.

White matter abnormalities were detected in 4 fetuses with CHD and in none of the control fetuses. These abnormalities included T2-weighted signal abnormality and cysts in the white matter. Acquired white matter signal hyperintensity was exclusively found in 2 fetuses with transposition of the great arteries. Subependymal cysts (possibly the residua of ischemic-hemorrhagic injury) were present in 2 fetuses, one with transposition of the great arteries and another with tetralogy of Fallot. Although the available literature is limited, the prognosis of isolated subependymal cysts diagnosed in utero has been good. ${ }^{18,19}$ White matter abnormalities, thought to be a form of periventricular leukomalacia, have been reported in fetuses with HLHS $^{20}$ on postmortem examination and in a number of postnatal studies of infants with CHD. ${ }^{5,21,22}$ The relation between the fetal WM abnormalities seen in our population and the periventricular leukomalacia described after birth in infants with CHD is not clear but could support an antenatal onset in some cases. The prognostic significance of fetal white matter signal intensity in this population remains to be determined. The current limitations of conventional fetal MR imaging may have hindered our ability to detect other cases of white matter abnormalities in utero. Although diffusion-weighted imaging is increasingly used in fetal MR imaging, this acquisition is particularly sensitive to fetal motion that results in image degradation. ${ }^{23} \mathrm{~A}$ recent case report suggested that differences in the white matter microstructural organization could also be detected in utero during the third trimester of 3 fetuses with CHD compared with healthy fetuses. ${ }^{24}$ However, further studies that assess white matter integrity in fetuses with CHD are needed to better understand the relationship between these macro- and microstructural preliminary findings and their long-term consequences.

In postnatal preoperative studies of infants with $\mathrm{CHD}$, the most prevalent brain lesions included ischemic lesions ${ }^{21}$ and diffuse and focal white matter injury, ${ }^{6,25,26}$ including periventricular leukomalacia. ${ }^{5,27}$ The brain MR imaging findings in our fetal cohort with CHD differed in both type and severity from these postnatal studies of infants with CHD. In fact, with the exception of 1 fetus with diffuse white matter injury, the brain anomalies described in our sample were all mild in severity. This difference in lesion severity could be explained, in part, by hemodynamic insults occurring during the complex transition from a fetal to postnatal circulation, a potentially hazardous period for infants with $\mathrm{CHD}^{28}$ Moreover, recent evidence suggests that brain injuries found in neonates with CHD preoperatively are associated with 
microstructural and metabolic markers of brain immaturity. ${ }^{29}$ Follow-up postnatal MR imaging studies and neurodevelopmental outcome assessments are needed to determine whether the prenatal findings described herein are transient or persistent in nature and their impact on long-term outcome. These studies are currently underway.

To our knowledge, this study is the first prospective study to describe conventional clinical MR imaging findings in a large sample of fetuses with CHD during the second and third trimesters of pregnancy and to compare them with a large control group of fetuses from healthy pregnancies. Moreover, our CHD sample included a representative range of cardiac diagnostic categories and was not limited to those with the most severe forms of CHD (eg, HLHS). However, given that we carefully excluded cases with multiorgan dysmorphic conditions, as well as chromosomal or genetic conditions, we may have underestimated the true prevalence of brain abnormalities in the overall fetal CHD population. Structural abnormalities of the brain may result as part of a spectrum of other congenital malformations including CHD. Finally, our overall goal was to describe the prevalence and spectrum of brain abnormalities detected on conventional MR imaging in fetuses with complex CHD. This objective was carried out by determining the frequency with which neuroradiologists made the clinical diagnosis of brain abnormality in fetuses with CHD compared with healthy control fetuses. Therefore, we did not perform repeat blinded MR imaging interpretations to assess intra- and inter-reader reliability. Nevertheless, our study was conducted in 2 major referral centers for congenital heart disease, and all MR imaging studies were reviewed by an experienced fetal neuroradiologist following clinical best practice standards. Consequently, we are confident that the findings reported in this study are representative of the current findings in clinical settings.

\section{CONCLUSIONS}

During the past decade, in vivo fetal MR imaging has contributed enormously to our understanding of fetal brain abnormalities in the living fetus. Our findings demonstrate that structural brain abnormalities are much more prevalent in fetuses with complex CHD than in controls and are not confined to those with the most critical forms of CHD.

However, although prevalent, the findings in our study were relatively mild in extent and were often suggestive of delayed or arrested brain maturation. These data contribute to a growing body of evidence suggesting that an important component of neurologic dysfunction in survivors of CHD is prenatal in origin.

Disclosures: Dorothy Bulas—RELATED: Grant: Canadian Institutes of Health Research.* UNRELATED: Consultancy: unpaid consultant to GE Healthcare and Philips Healthcare on ultrasound technology not relevant to this research, Royalties: UpToDate chapter royalties. Mary Donofrio-RELATED: Grant: Canadian Institutes of Health Research, ${ }^{*}$ Catherine Limperopoulos—RELATED: Grant: Canadian Institutes of Health Research.* *Money paid to the institution.

\section{REFERENCES}

1. Owen M, Shevell M, Majnemer A, et al. Abnormal brain structure and function in newborns with complex congenital heart defects before open heart surgery: a review of the evidence. J Child Neurol 2011;26:743-55
2. Donofrio MT, Massaro AN. Impact of congenital heart disease on brain development and neurodevelopmental outcome. Int J Pediatr 2010;2010. pii:359390

3. Block AJ, McQuillen PS, Chau V, et al. Clinically silent preoperative brain injuries do not worsen with surgery in neonates with congenital heart disease. J Thorac Cardiovasc Surg 2010;140:550-57

4. Licht DJ, Shera DM, Clancy RR, et al. Brain maturation is delayed in infants with complex congenital heart defects. $J$ Thorac Cardiovasc Surg 2009;137:529-36, discussion 536-37

5. Licht DJ, Wang J, Silvestre DW, et al. Preoperative cerebral blood flow is diminished in neonates with severe congenital heart defects. J Thorac Cardiovasc Surg 2004;128:841-49

6. Miller SP, McQuillen PS, Hamrick S, et al. Abnormal brain development in newborns with congenital heart disease. $N$ Engl J Med 2007;357:1928-38

7. Limperopoulos C, Tworetzky W, McElhinney DB, et al. Brain volume and metabolism in fetuses with congenital heart disease: evaluation with quantitative magnetic resonance imaging and spectroscopy. Circulation 2010;121:26-33

8. Clouchoux C, du Plessis AJ, Bouyssi-Kobar M, et al. Delayed cortical development in fetuses with complex congenital heart disease. Cereb Cortex 2013;23:2932-43

9. Mlczoch E, Brugger P, Ulm B, et al. Structural congenital brain disease in congenital heart disease: results from a fetal MRI program. Eur J Paediatr Neurol 2013;17:153-60

10. Wax JR, Bookman L, Cartin A, et al. Mild fetal cerebral ventriculomegaly: diagnosis, clinical associations, and outcomes. Obstet Gynecol Surv 2003;58:407-14

11. Girard NJ, Raybaud CA. Ventriculomegaly and pericerebral CSF collection in the fetus: early stage of benign external hydrocephalus? Childs Nerv Syst 2001;17:239-45

12. Watanabe $Y$, Abe $S$, Takagi K, et al. Evolution of subarachnoid space in normal fetuses using magnetic resonance imaging. Prenat Diagn 2005;25:1217-22

13. Glenn OA, Barkovich AJ. Magnetic resonance imaging of the fetal brain and spine: an increasingly important tool in prenatal diagnosis, part 1. AJNR Am J Neuroradiol 2006;27:1604-11

14. Donofrio MT. The heart-brain interaction in the fetus: cerebrovascular blood flow in the developing human. Prog Pediatr Cardiol 2006;22:41-51

15. Donofrio MT, Bremer YA, Schieken RM, et al. Autoregulation of cerebral blood flow in fetuses with congenital heart disease: the brain sparing effect. Pediatr Cardiol 2003;24:436-43

16. Fukui K, Morioka T, Nishio S, et al. Fetal germinal matrix and intraventricular haemorrhage diagnosed by MRI. Neuroradiology 2001;43:68-72

17. Tarui T, Limperopoulos C, Sullivan NR, et al. Long-term developmental outcome of children with a fetal diagnosis of isolated inferior vermian hypoplasia. Arch Dis Child Fetal Neonatal Ed 2014;99:F54-58

18. Bats AS, Molho M, Senat MV, et al. Subependymal pseudocysts in the fetal brain: prenatal diagnosis of two cases and review of the literature. Ultrasound Obstet Gynecol 2002;20:502-05

19. D’Addario V, Selvaggio S, Pinto V, et al. Fetal subependymal cysts with normal neonatal outcome: a case report. Fetal Diagn Ther 2003; 18:170-73

20. Hinton RB, Andelfinger G, Sekar P, et al. Prenatal head growth and white matter injury in hypoplastic left heart syndrome. Pediatr Res 2008;64:364-69

21. Mahle WT, Tavani F, Zimmerman RA, et al. An MRI study of neurological injury before and after congenital heart surgery. Circulation 2002;106(12 suppl 1):1109-14

22. Andropoulos DB, Hunter JV, Nelson DP, et al. Brain immaturity is associated with brain injury before and after neonatal cardiac surgery with high-flow bypass and cerebral oxygenation monitoring. J Thorac Cardiovasc Surg 2010;139:543-56

23. Clouchoux C, Limperopoulos C. Novel applications of quantitative MRI for the fetal brain. Pediatr Radiol 2012;42(suppl 1):S24-32 
24. Berman JI, Hamrick SEG, McQuillen PS, et al. Diffusion-weighted imaging in fetuses with severe congenital heart defects. AJNR Am J Neuroradiol 2011;32:E21-22

25. McQuillen PS, Hamrick SE, Perez MJ, et al. Balloon atrial septostomy is associated with preoperative stroke in neonates with transposition of the great arteries. Circulation 2006;113:280-85

26. Partridge SC, Vigneron DB, Charlton NN, et al. Pyramidal tract maturation after brain injury in newborns with heart disease. Ann Neurol 2006;59:640-51
27. Petit CJ, Rome JJ, Wernovsky G, et al. Preoperative brain injury in transposition of the great arteries is associated with oxygenation and time to surgery, not balloon atrial septostomy. Circulation 2009;119:709-16

28. Friedman AH, Fahey JT. The transition from fetal to neonatal circulation: normal responses and implications for infants with heart disease. Semin Perinatol 1993;17:106-21

29. Dimitropoulos A, McQuillen PS, Sethi V, et al. Brain injury and development in newborns with critical congenital heart disease. $\mathrm{Neu}$ rology 2013;81:241-48 